\title{
Utilización de Residuos Forestales Lignocelulósicos para Producción del Hongo Ostra (Pleurotus ostreatus)
}

María T. Varnero ${ }^{1}$, Madelaine S. Quiroz ${ }^{1}$ y Cristian H. Álvarez ${ }^{2}$

(1) Universidad de Chile, Facultad de Ciencias Agronómicas, Casilla 1004, Santiago-Chile

(e-mail: mvarnero@uchile.cl, madquiroz@gmail.com)

(2) Ministerio del Trabajo y Previsión Social, Huérfanos 1273 Santiago-Chile

(e-mail: transparencia@mintrab.gob.cl)

\section{Resumen}

Se estudió el potencial de distintos residuos forestales como sustrato para el cultivo del hongo Pleurotus ostreatus. Para ello, se analizó la composición química de estos residuos, antes y después de la etapa de cosecha, y se midieron distintas variables fenológicas y morfológicas así como el rendimiento y la calidad de los cuerpos fructíferos de Pleurotus ostreatus tras la cosecha. Se fijaron cuatro tratamientos: astillas de álamo, astillas de eucalipto, mezcla de paja de trigo y eucalipto, y paja de trigo como testigo. Los resultados obtenidos indicaron que todos los sustratos, principalmente paja de trigo y mezcla paja de trigo más eucalipto son aptos para el cultivo de Pleurotus ostreatus. El nivel proteico de este hongo fue elevado en todos los sustratos y la relación carbono/nitrógeno de los mismos disminuyó después de cosecha.

Palabras clave: hongo ostra, residuos forestales, residuos lignocelulósicos, sustratos

\section{Use of Lignocellulosic Forest Residues for Oyster Mushroom Production (Pleurotus ostreatus)}

\begin{abstract}
Organic waste resulting from the forest practices, containing lignocellulosic carbon sources, was used in the production of the mushroom specie Pleurotus ostreatus. For this, the chemical composition before and after harvest were analyzed, the phenological and morphological variables were determined and yield and quality of harvested fruit bodies also determined. Four treatments were performed: chips of poplars, chips of eucalyptus, a mixture of wheat straw and eucalyptus, and wheat straw as witness. The results showed that all substrates, especially wheat straw and wheat straw with eucalyptus, are suitable for the production of Pleurotus ostreatus. This species showed a high protein level in all the studied substrates, and the ratio carbon/nitrogen decreased after harvest.
\end{abstract}

Keywords: oyster mushroom, forest residues, lignocellulosic wastes, substrates 


\section{INTRODUCCIÓN}

En los últimos años los principales países productores de madera han centrado su interés en la búsqueda de alternativas para la gestión y la utilización de los residuos derivados de las actividades forestales. Las alternativas de aprovechamiento implementadas hasta el momento se han enfocado fundamentalmente hacia su recuperación energética (Cerda, 1998). Sin embargo, considerando su alto contenido en fuentes de carbono lignocelulósicas (Bonatti et al., 2004), resultaría interesante explorar las posibilidades de uso de estos residuos como sustrato para el cultivo y producción de hongos comestibles. La crisis energética, el deterioro del medio ambiente y el acusado crecimiento demográfico ponen de manifiesto la importancia de producir alimentos proteicos de calidad, siempre que su producción no ocasione un riesgo contaminante. Los hongos comestibles constituyen una importante fuente de alimento, y algunos países en vías de desarrollo como Chile los consideran una alternativa novedosa para la obtención de alimentos de bajo costo. Pleurotus ostreatus es la tercera seta de mayor comercialización a nivel internacional y la segunda a nivel nacional, estimándose un mercado en crecimiento para los próximos años, debido, en parte, a que su cultivo se desarrolla mayoritariamente en paja de trigo, residuo que se encuentra disponible en el centro y sur del país (Medina y Cisterna, 2002). Por otra parte, la utilización de residuos de origen maderero como sustrato para el cultivo de este hongo también ayuda al aprovechamiento de productos del bosque nativo (Orensanz y Navarro, 1979).

$P$. ostreatus es un hongo lignícola saprófito, conocido con el nombre común de hongo ostra. Pertenece a la clase Basidiomicetes, orden Agaricales, familia Agaricacea (Donoso y Aguirre, 1980; Furci, 2007). En condiciones silvestres crece en tocones y ramas de planifolios muertos o debilitados, en bosques de rivera, parques y jardines (Alpuche y Paredes, 1996). Su desarrollo ocurre durante la estación otoñal e inicio de primavera, aunque en sitios húmedos también es posible encontrarlo en otras estaciones del año. Esta especie presenta gran versatilidad y adaptabilidad, ya que tolera un rango amplio de temperaturas; además, presenta resistencia a plagas y enfermedades, y se puede cultivar prácticamente sobre cualquier sustrato lignocelulósico como troncos, corteza o aserrín (García, 1987; Levin, 1996; Adamovic et al., 1998; Yildiz et al., 2002). En Chile, esta especie se encuentra en los bosques de la zona centro sur, sobre tocones de sauces, álamos y castaños (INFOR y Fundación Chile, 2008).

La composición química de $P$. ostreatus es muy variable y depende del estado de desarrollo y la cepa utilizada; la variabilidad es ocasionada por diferencias en el contenido de humedad, temperatura y la presencia de nutrientes. Esta especie constituye un alimento altamente proteico, posee un elevado contenido de vitaminas (Tiamina (B1), Riboflavina (B2), Piridoxina (B6) y Cobalamina (B12)); y actúa como una fuente importante de calcio y fósforo (Breene, 1990). Además de su valor nutritivo, $P$. ostreatus posee enzimas específicas capaces de degradar lignina, fenoles y polifenoles hasta un $60 \%$ del contenido original, por lo que es una de las especies más utilizadas en la investigación de residuos aptos para su cultivo (Espinoza, 1997). Normalmente, el cultivo de Pleurotus se realiza sobre sustratos preparados con paja de cereales, como trigo, centeno o cebada, cortados en trozos de 2 a $4 \mathrm{~cm}$ de longitud. A modo de ejemplo, se ha observado una producción de 100 a $200 \mathrm{~kg}$ por tonelada de paja de trigo (García, 1987). Yildiz et al (2002) y Salmones et al. (2005), también observaron una elevada producción de $P$. ostreatus en residuos derivados de actividades agroindustriales, como pulpa de café, hojas usadas en la extracción de aceites esenciales y bagazo de caña de azúcar.

El objetivo de este estudio fue evaluar el potencial de distintos residuos forestales como sustrato para el cultivo de este hongo. Se analizó la composición química de los residuos antes y después de la etapa de cosecha, y se determinaron las propiedades fenológicas, morfológicas, rendimiento y calidad de los cuerpos fructíferos de $P$. ostreatus tras la cosecha.

\section{MATERIALES Y MÉTODO}

\section{Preparación del inóculo}

El hongo $P$. ostreatus se obtuvo de troncos muertos de álamo (Populus sp.), en la localidad de Santa Cruz, ubicada a $40 \mathrm{Km}$ de la ciudad de San Fernando, Chile. El micelio se propagó en placas Petri 
colocando $1 \mathrm{~g}$ de micelio en $5 \mathrm{ml}$ de agar papa dextrosa y se inoculó en un medio de cultivo elaborado con granos de trigo previamente esterilizados en frascos de vidrio de $250 \mathrm{~g}$.

\section{Sustratos empleados para el cultivo de P. ostreatus}

Se utilizaron los siguientes residuos lignocelulósicos: paja de trigo (Triticum aestivum L.), astillas de eucalipto (Eucalytus globulus); y astillas de álamo (Populus nigra), las cuales presentaron una longitud aproximada de $2 \mathrm{~cm}$. Previo a la inoculación de Pleurotus ostreatus, estos sustratos se sumergieron en agua a $70^{\circ} \mathrm{C}$ durante 60 minutos. Se establecieron los siguientes tratamientos experimentales con cinco réplicas por cada tratamiento (Tabla 1):

Tabla 1: Tratamientos usados para la evaluación del cultivo de Pleurotus ostreatus

\begin{tabular}{|c|l|}
\hline Tratamientos & \multicolumn{2}{|c|}{} \\
\hline $\mathrm{T} 1$ & $1 \mathrm{~kg}$ paja de trigo $+50 \mathrm{~g}$ de grano invadido con micelio. \\
\hline $\mathrm{T} 2$ & $1 \mathrm{~kg}$ astillas de eucalipto $+50 \mathrm{~g}$ de grano invadido con micelio. \\
\hline $\mathrm{T} 3$ & $150 \mathrm{~g}$ paja de trigo $+850 \mathrm{~g}$ astillas de eucalipto $+50 \mathrm{~g}$ de grano invadido con micelio. \\
\hline $\mathrm{T} 4$ & $1 \mathrm{~kg}$ astilla de álamo $+50 \mathrm{~g}$ de grano invadido con micelio. \\
\hline
\end{tabular}

\section{Siembra e incubación}

Los sustratos se inocularon con 5\% de micelio del hongo crecido en granos de trigo previamente hidratados y esterilizados. La siembra se hizo en bolsas plásticas, las cuales se perforaron para promover una condición de semi-anaerobiosis requerida en la fase inicial de invasión del micelio, y los tratamientos se incubaron a $25^{\circ} \mathrm{C}$ con baja luminosidad (inferior a 100 lux). Una vez producida la invasión total del micelio en los sustratos (25 a 30 días), las bolsas se dejaron a temperatura ambiente $\left(15^{\circ} \mathrm{C}\right.$ aproximadamente) y luminosidad normal (200 lux) durante 12 días; de este modo se consiguió estimular la brotación del hongo exponiendo los sustratos a un golpe de frío. Dado que esta fase es aeróbica, se retiraron las bolsas plásticas, lo que permitió mantener la forma inicial del recipiente conformando una torre. Los sustratos se regaron periódicamente para mantener una humedad cercana al $70 \%$.

\section{Etapa de cosecha}

La cosecha de los cuerpos fructíferos se realizó cuando los sombreros alcanzaron $3 \mathrm{~cm}$ de diámetro en los ejemplares menores y antes que los ejemplares mayores cambiaran su forma convexa a cóncava. Los racimos se arrancaron por completo para evitar infecciones. El período de cosecha concluyó después de 30 días, una vez realizada la cosecha del último racimo, sin la aparición de nuevos primordios.

\section{Análisis}

Se midieron variables químicas de los sustratos al inicio y término del ensayo (TMECC, 2002): contenido de humedad, $\mathrm{pH}$, materia orgánica, nitrógeno $(\mathrm{N})$, fósforo $(\mathrm{P})$ y potasio $(\mathrm{K})$ total y relación carbono/nitrógeno $(\mathrm{C} / \mathrm{N})$.

Se determinaron variables fenológicas y morfológicas de Pleurotus ostreatus, así como rendimiento y calidad de los hongos. Se determinó la eficiencia biológica (EB), como porcentaje de producción de hongos peso fresco con relación a peso seco de sustrato (Mandeel et al., 2005). El contenido proteico se determinó desde el contenido de nitrógeno total medido en los hongos, usando el factor 4,38 (Breene, 1990).

\section{Análisis estadístico}

El diseño experimental correspondió a bloques completos al azar con cinco réplicas por tratamiento. La unidad experimental correspondió a una torre, forma que adquiere el sustrato contenido en la bolsa de plástico. Los datos se analizaron mediante un análisis de varianza o ANDEVA $(P=0,05), y$ 
las diferencias entre tratamientos se determinaron utilizando el test de comparaciones múltiples de Duncan $(P=0,05)$.

\section{RESULTADOS Y DISCUSIÓN}

\section{Composición química de los sustratos utilizados para el cultivo de P. ostreatus}

En la etapa de precosecha, se observaron diferencias significativas entre tratamientos con respecto al contenido de $\mathrm{N}_{\mathrm{T}}, \mathrm{P}_{\mathrm{T}}, \mathrm{K}_{\mathrm{T}}$ (Tabla 2, ANDEVA $\mathrm{P}=0$,05). Los resultados del test de Duncan indican que el contenido de estos nutrientes fue significativamente mayor en el tratamiento de paja de trigo, evidenciándose también, su aporte nitrogenado a la mezcla paja-eucalipto. Por otra parte, los valores nutricionales más bajos se registraron en los tratamientos de eucalipto y álamo, los cuales contienen altos niveles de fracciones lignocelólusicas, lo que podría explicar los menores valores promedios de $\mathrm{N}_{\mathrm{T}}$ presentados (0,6 g y 0,46 g respectivamente). En la etapa de post cosecha, el tratamiento paja de trigo también presentó los valores más altos (Tabla 3). Al comparar ambas etapas, se observó un aumento en el contenido de $\mathrm{N}_{\mathrm{T}}$ de todos los sustratos y una reducción en la concentración de $\mathrm{K}_{\mathrm{T}}$.

Tabla 2: Variables químicas de los sustratos en pre cosecha utilizados en el ensayo (valores promedios en base masa seca). Letras minúsculas diferentes indican diferencias estadísticamente significativas entre tratamientos con un $\mathrm{P}=0,05$. * indica diferencias estadísticamente significativas entre precosecha y post cosecha con un $\mathrm{P}=0,05$.

\begin{tabular}{|l|c|c|c|c|c|c|}
\hline Sustratos Precosecha & $\begin{array}{c}\mathrm{N}_{\mathrm{T}} \\
\mathrm{g} / \mathrm{kg}\end{array}$ & $\begin{array}{c}\mathrm{P}_{\mathrm{T}} \\
\mathrm{g} / \mathrm{kg}\end{array}$ & $\begin{array}{c}\mathrm{K}_{\mathrm{T}} \\
\mathrm{g} / \mathrm{kg}\end{array}$ & $\begin{array}{c}\mathrm{MO} \\
\mathrm{g} / \mathrm{kg}\end{array}$ & $\mathrm{C} / \mathrm{N}$ & $\mathrm{pH}$ \\
\hline Paja de Trigo & $3,64 \mathrm{a}^{*}$ & $0,11 \mathrm{a}$ & $1,82 \mathrm{a}^{*}$ & $920 \mathrm{~d}^{*}$ & $147 \mathrm{~d}^{*}$ & $4,4 \mathrm{~b}$ \\
\hline Eucalipto & $0,60 \mathrm{c}^{*}$ & $0,02 \mathrm{c}^{*}$ & $0,15 \mathrm{~d}^{*}$ & $994 \mathrm{a}$ & $1009 \mathrm{~b}^{*}$ & $3,7 \mathrm{c}^{*}$ \\
\hline Paja - Eucalipto & $1,06 \mathrm{~b}^{\star}$ & $0,03 \mathrm{~b}$ & $0,40 \mathrm{~b}^{*}$ & $983 \mathrm{bc}$ & $545 \mathrm{c}^{*}$ & $3,8 \mathrm{c}$ \\
\hline Álamo & $0,46 \mathrm{c}^{*}$ & $0,01 \mathrm{~d}$ & $0,29 \mathrm{c}^{*}$ & $986 \mathrm{~b}$ & $1277 \mathrm{a}^{*}$ & $5,5 \mathrm{a}^{*}$ \\
\hline
\end{tabular}

Tabla 3: Variables químicas de los sustratos en post cosecha utilizados en el ensayo (valores promedios en base masa seca). Letras minúsculas diferentes indican diferencias estadísticamente significativas entre tratamientos con un $\mathrm{P}=0,05$. * indica diferencias estadísticamente significativas entre precosecha y post cosecha con un $\mathrm{P}=0,05$.

\begin{tabular}{|l|c|c|c|c|c|c|}
\hline $\begin{array}{l}\text { Sustratos } \\
\text { Postcosecha }\end{array}$ & $\begin{array}{c}\mathrm{N}_{\mathrm{T}} \\
\mathrm{g} / \mathrm{kg}\end{array}$ & $\begin{array}{c}\mathrm{P}_{\mathrm{T}} \\
\mathrm{g} / \mathrm{kg}\end{array}$ & $\begin{array}{c}\mathrm{K}_{\mathrm{T}} \\
\mathrm{g} / \mathrm{kg}\end{array}$ & $\begin{array}{c}\mathrm{MO} \\
\mathrm{g} / \mathrm{kg}\end{array}$ & $\mathrm{C} / \mathrm{N}$ & $\mathrm{pH}$ \\
\hline Paja de Trigo & $6,86 \mathrm{a}^{*}$ & $0,13 \mathrm{a}$ & $0,96 \mathrm{a}^{\star}$ & $893 \mathrm{c}^{*}$ & $77^{\star} \mathrm{d}$ & $5,3 \mathrm{a}$ \\
\hline Eucalipto & $0,84 \mathrm{c}^{\star}$ & $0,01 \mathrm{c}^{\star}$ & $0,03 \mathrm{c}^{\star}$ & $991 \mathrm{a}$ & $692 \mathrm{ab}^{\star}$ & $3,5 \mathrm{~d}^{\star}$ \\
\hline Paja - Eucalipto & $1,68 \mathrm{~b}^{\star}$ & $0,03 \mathrm{~b}$ & $0,06 \mathrm{bc}^{\star}$ & $922 \mathrm{abc}$ & $325 \mathrm{c}^{\star}$ & $4,0 \mathrm{bc}$ \\
\hline Álamo & $0,84 \mathrm{c}^{\star}$ & $0,02 \mathrm{c}$ & $0,07 \mathrm{~b}^{*}$ & $985 \mathrm{ab}$ & $751 \mathrm{a}^{\star}$ & $5,1 \mathrm{ab}^{\star}$ \\
\hline
\end{tabular}

En precosecha, respecto al contenido de materia orgánica, el sustrato de eucalipto presentó el mayor valor, presentando diferencias significativas con los sustratos de álamo y paja de trigo; por el contrario, este último presentó el menor valor de contenido de MO y se observaron diferencias estadísticas significativas con el sustrato paja de trigo-eucalipto (Tabla 2). En post cosecha estas diferencias no fueron concluyentes, observándose diferencias estadísticas significativas sólo en los sustratos eucalipto y paja de trigo (Tabla 3). Al comparar los valores entre tratamientos en pre y post cosecha se observó que todos los sustratos presentaron una disminución del contenido de MO al final del ensayo, pero sólo presentó diferencias estadísticamente significativas el sustrato con paja de trigo.

En cuanto a la relación $\mathrm{C} / \mathrm{N}$, en precosecha, se apreciaron diferencias estadísticas significativas entre todos los tratamientos (Tabla 2), presentando el valor más alto el sustrato compuesto por álamo, lo cual puede atribuirse al mayor contenido de fracciones lignocelulósicas de este residuo, siendo el sustrato con paja de trigo el que presentó el menor valor de relación C/N. Igual tendencia se observó 
en post cosecha (Tabla 3). Al comparar ambas etapas, se observó una disminución de la relación $\mathrm{C} / \mathrm{N}$ en todos los tratamientos existiendo diferencias estadísticas significativas para cada tratamiento en pre y post cosecha. Este resultado se explicaría por el incremento relativo de $\mathrm{N}_{\mathrm{T}}$ en los distintos sustratos, como consecuencia de la disminución del contenido de carbono en forma de desprendimiento de dióxido de carbono $\left(\mathrm{CO}_{2}\right)$. Esto último, estaría asociado a la actividad microbiológica desarrollada en el interior de los sustratos y, principalmente, por el uso de fuentes de carbono presentes en éstos para el desarrollo y producción de hongos.

Los valores de $\mathrm{pH}$ observados en precosecha (Tabla 2), fluctuaron en el rango de $\mathrm{pH}$ ácido observándose diferencias significativas entre sustratos, presentando el sustrato eucalipto el menor valor. Similar tendencia se observó en la etapa de post cosecha. (Tabla 3). Al comparar los pares de sustratos en ambas etapas, sólo se observaron diferencias estadísticas significativas con álamo y eucalipto.

\section{Descripción de las variables fenológicas y morfológicas de P. ostreatus}

Las variables fenológicas de $P$. ostreatus, medidas en los diferentes sustratos (Tabla 4), señalan que en el período de Siembra-Primordio, el sustrato paja de trigo requirió el mayor número de días para desarrollar primordios, presentando diferencias estadísticas significativas respecto de los demás tratamientos, en cambio los sustrato eucalipto y álamo presentaron el menor tiempo en el desarrollo de primordios, lo cual podría explicarse por la forma de propagación del micelio. En el caso del sustrato con álamo la invasión del micelio fue parcial, concentrándose en la base de la torre y en eucalipto presentó mayor desarrollo en la parte media y superior de esta. En cambio, en el sustrato paja de trigo y mezcla paja de trigo-eucalipto, la invasión fue prácticamente total, de este modo el tiempo de propagación fue mayor produciéndose un retraso en la emergencia de primordios.

En el período de Primordio-Cosecha (Tabla 4), el sustrato eucalipto presentó mayor tiempo de inicio de cosecha existiendo diferencias estadísticas significativas con los otros tratamientos, en cambio el sustrato álamo presentó menor tiempo para el inicio de cosecha. Posiblemente estos resultados estarían relacionados con las características bioquímicas de los distintos sustratos, que podrían hacer variar el período Primordio-Cosecha, permitiendo una disminución en este período en algunos casos.

Tabla 4: Variables fenológicas de $P$. ostreatus. Letras minúsculas diferentes indican diferencias estadísticamente significativas entre tratamientos en cada período, $\mathrm{P}=0,05$.

\begin{tabular}{|l|c|c|c|c|}
\hline \multicolumn{1}{|c|}{ Tratamientos } & \multicolumn{4}{|c|}{ Valores promedios para cada período fenológico (días) } \\
\hline Primordio & $\begin{array}{c}\text { Primordio - } \\
\text { Cosecha }\end{array}$ & $\begin{array}{c}\text { Período de } \\
\text { cosecha }\end{array}$ & $\begin{array}{c}\text { Siembra - Final de } \\
\text { Cosecha }\end{array}$ \\
\hline Eaja de trigo & $69,0 \mathrm{a}$ & $10,4 \mathrm{c}$ & $25,2 \mathrm{a}$ & $104,6 \mathrm{a}$ \\
\hline Pucalipto & $41,4 \mathrm{c}$ & $14,8 \mathrm{a}$ & $15,0 \mathrm{c}$ & $71,2 \mathrm{c}$ \\
\hline Álamo & $51,4 \mathrm{~b}$ & $13,6 \mathrm{~b}$ & $20,6 \mathrm{~b}$ & $85,6 \mathrm{~b}$ \\
\hline
\end{tabular}

El sustrato paja de trigo presentó el mayor periodo de cosecha (Tabla 4), y el menor periodo lo presentó el sustrato álamo. Estos resultados podrían explicarse debido a la forma de invasión del micelio, así en sustratos con mayor propagación del hongo, se favorecería la obtención de cosechas prolongadas, lo cual estaría asociado al rendimiento final del cultivo. Orensanz y Navarro (1979), señalan que aquellas maderas con mayor densidad presentan mayores períodos de cosecha que maderas con menor densidad. En este caso, al comparar el período de cosecha de los sustratos de eucalipto y álamo, se observó esta situación, ya que la madera de eucalipto presenta mayor densidad que la madera de álamo.

Los sustratos en base a cereales, han sido habitualmente utilizados por sus altos rendimientos y según lo reportado por García, 1987, aquel sustrato elaborado en base a paja de trigo presentaría los mayores rendimientos para el hongo, lo cual coincide con los resultados obtenidos en este trabajo. Por otra parte, se observa que el período de cosecha de la mezcla paja de trigo-eucalipto presenta 
mayor duración que el sustrato eucalipto, lo cual podría atribuirse a la proporción incorporada de paja de trigo, la que aumentaría el periodo de cosecha en la mezcla paja de trigo-eucalipto.

Esta situación se verifica cuando se analiza el período de Siembra-Final de Cosecha (Tabla 4), donde se apreciaron diferencias estadísticamente significativas entre todos los sustratos. El período de mayor duración lo presentó el sustrato paja de trigo, seguido de la mezcla paja de trigo-eucalipto, eucalipto y, finalmente álamo. Por lo que se ha establecido una relación entre la duración del período Siembra-Final de Cosecha y el rendimiento.

La forma y color de los hongos resultaron similares para todos los sustratos. Sin embargo, la consistencia fue superior en los sustratos con mayor rendimiento.

Los sustratos paja de trigo y mezcla paja de trigo-eucalipto produjeron el mayor número de hongos (Tabla 5) y el menor número se observó en el sustrato álamo. Una situación similar ocurrió con el número de racimos, lo cual se podría atribuir a la forma de invasión del micelio en la torre, como se mencionó anteriormente. Así, una mayor cantidad de racimos se podría atribuir al mayor desarrollo o propagación del micelio en los sustratos. Finalmente, respecto al número de hongos por racimo el sustrato paja de trigo presentó un equilibrio entre la cantidad de hongos y sus correspondientes racimos, existiendo diferencias estadísticas significativas con los demás tratamientos.

Tabla 5: Variables morfológicas de $P$. ostreatus. Letras minúsculas diferentes indican diferencias estadísticamente significativas entre tratamientos $P=0,05$.

\begin{tabular}{|c|c|c|c|}
\hline Tratamientos & Número de hongos & Número de racimos & Número de hongos/racimo \\
\hline Paja de Trigo & $18,2 \mathrm{a}$ & $5,2 \mathrm{ab}$ & $3,4 \mathrm{a}$ \\
\hline Eucalipto & $10,2 \mathrm{bc}$ & $4,6 \mathrm{bc}$ & $2,2 \mathrm{~b}$ \\
\hline Paja/Eucalipto & $15,2 \mathrm{ab}$ & $6,8 \mathrm{a}$ & $2,3 \mathrm{~b}$ \\
\hline Álamo & $3,4 \mathrm{~d}$ & $2,8 \mathrm{~d}$ & $1,2 \mathrm{c}$ \\
\hline
\end{tabular}

Rendimiento de la producción de P. ostreatus

Se encontró que en la variable Peso Fresco Unitario de hongo en el cultivo (Tabla 6), no existieron diferencias estadísticamente significativas entre tratamientos, a pesar que en el sustrato paja de trigo se produjeron los hongos con mayor peso unitario. Lo anterior podría atribuirse a un factor genético de $P$. ostreatus, donde independientemente del sustrato utilizado para su cultivo, una vez que el hongo se desarrolla, su peso fresco no presentaría mayores variaciones. Similar situación se observó en el Peso Fresco Racimo, lo cual se explicaría por el mayor número de hongos que se obtiene en cada racimo, más que por el peso unitario de los hongos.

Al considerar el rendimiento del cultivo en términos de \%EB (Tabla 6), el mayor rendimiento se obtuvo en el sustrato paja de trigo existiendo diferencias significativas con los demás tratamientos. En cuanto al contenido de nitrógeno total y el nivel proteico de los hongos, obtenido en los diferentes sustratos, se aprecian valores similares y sin diferencias estadísticas significativas. Sin embargo, estos valores son superiores a los reportados por Bonatti et al., (2004) para $P$. ostreatus cultivados en sustratos de paja de arroz y de plátanos.

Tabla 6:. Variables de rendimiento de $P$. ostreatus. Letras minúsculas diferentes indican diferencias estadísticamente significativas entre tratamientos con un $\mathrm{P}=0,05$.

\begin{tabular}{|l|c|c|c|c|c|}
\hline Tratamientos & $\begin{array}{c}\text { Peso } \\
\text { fresco/unitario } \\
(\mathrm{g})\end{array}$ & $\begin{array}{c}\text { Peso } \\
\text { fresco/racimo } \\
(\mathrm{g})\end{array}$ & EB (\%) & $\begin{array}{c}\mathrm{N}_{\mathrm{T}} \\
\text { (peso seco) } \\
(\%)\end{array}$ & $\begin{array}{c}\text { Proteína total } \\
\text { (peso seco) } \\
(\%)\end{array}$ \\
\hline Paja de trigo & $18,1 \mathrm{a}$ & $55,1 \mathrm{a}$ & $32,94 \mathrm{a}$ & $5,85 \mathrm{a}$ & $25,6 \mathrm{a}$ \\
\hline Eucalipto & $4,2 \mathrm{a}$ & $8,6 \mathrm{bc}$ & $4,23 \mathrm{c}$ & $5,80 \mathrm{a}$ & $25,4 \mathrm{a}$ \\
\hline Paja/Eucalipto & $9,8 \mathrm{a}$ & $22 \mathrm{~b}$ & $14,93 \mathrm{~b}$ & $5,57 \mathrm{a}$ & $24,4 \mathrm{a}$ \\
\hline Álamo & $8,7 \mathrm{a}$ & $15,3 \mathrm{bc}$ & $2,97 \mathrm{c}$ & $5,22 \mathrm{a}$ & $22,9 \mathrm{a}$ \\
\hline
\end{tabular}




\section{CONCLUSIONES}

La producción o cultivo de $P$. ostreatus, sobre residuos lignocelulósicos es factible, constituyendo un proceso de bioconversión eficiente, el que se ve reflejado en el alto nivel proteico de las setas obtenidas en todos los tratamientos, y además, en la calidad de todos los sustratos utilizados para el cultivo, los cuales presentaron una disminución de la relación C/N una vez concluido el ensayo.

Estos materiales orgánicos podrían utilizarse como sustrato en producciones posteriores de $P$. ostreatus, incorporarse al suelo como acondicionador y/o mejorador de las propiedades físicas, o bien, constituir parte de mezclas elaboradas en la producción de sustratos para viveros.

\section{REFERENCIAS}

Adamovic, M., G. y otros seis autores. The biodegradation of wheat straw by Pleurotus ostreatus mushrooms and its use in cattle feeding. Animal Feed Science and Technology: 3-4 (71), 357-362 (1998)

Alpuche, G. y O. Paredes; Potencial en la biotecnología para la producción de hongos comestibles empleando desperdicios agrícolas. Acta Universitaria: México, 1(4), 1-15 (1996.)

Bonatti, M., P. Karnopp, H.M. Soares y S.A. Furlan; Evaluation of Pleurotus ostreatus and Pleurotus sajor-caju nutricional characteristic when cultivated in different lignocelululosic wastes. Food Chemistry: 88, 425-428 (2004).

Breene, W.M.; Nutritional and medicinal value of specialty mushrooms. Journal of Food Protection: 10(53), 883-894 (1990).

Cerda I.; Trayectoria del sector forestal chileno: Sus logros y desafíos. Instituto Forestal. Santiago, Chile, 3-91 (1998).

Donoso, J. y J. Aguirre; Cultivo de hongos comestibles lignívoros. Corporación de Fomento. Gerencia de Desarrollo, 4-70 (1980).

Espinoza, R.M.; Podría utilizarse la celulosa de pañales desechables para el cultivo de hongos comestibles. Vida Universitaria: Azcapotzalco, México: 12, 1-2 (1997)

Furci G.; Fungi Austral: Guía de campo de los hongos más vistosos de Chile. Ed. Corporación Chilena de la Madera. Concepción, Chile, 1-200 (2007).

García M.; Cultivo de setas y trufas. Editorial Mundi. Madrid, España, 2-255 (1987)

INFOR y Fundación Chile. Productos forestales no madereros (2008). Disponible en: http://www.gestionforestal.cl:81/pfnm/index.htm. Acceso: 3 de marzo 2008.

Levin, L.; Biodegradación de materiales lignocelulósicos por hongos. Secretaría de Ciencias y Técnica. Universidad de Buenos Aires, Argentina, 1-20 (1996)

Mandeel, Q.A., A.A. Al-Laith y S.A. Mohamed; Cultivation of oyster muhsrooms (Pleurotus spp.) on various lignocellulosic wastes. World Journal of Microbiology \& Biotecnology: 21, 601:607 (2005).

Medina R. y C. Cisterna; Hongos comestibles (2002) Disponible en: http://www.micotec.cl/ oportunidades.html. Micotec Ltda. Acceso: 15 de enero de 2008.

TMECC. Test Methods for Examination of Composting and Compost U.S Composting Council Research and education Foundation. (2002). Disponible: http://www.tmecc.org. Acceso:30 de Julio de 2006.

Orensanz, J. y C. Navarro; Cultivo del Pleurotus ostreatus sobre madera. Hojas Divulgadoras №3. Ministerio de Agricultura, Pesca y Alimentación. Madrid, España, 1-20 (1979) 
Salmones, D., G. Mata y K. N. Waliszewski; Comparative culturing of Pleurotus spp. on coffee pulp and wheat straw: biomass production and substrate biodegradation. Bioresourse Technology: 5(96) 537-544 (2005)

Yildiz, S., U.C. Yildiz, E.D. Gezer y A. Temiz; Some lignocellulosic wastes used as raw material in cultivation of the Pleurotus ostreatus culture mushroom. Process Biochemistry: 3(38), 301-306 (2002) 